\title{
HETEROGENEIDADE DAS EXPECTATIVAS DE INFLAÇÃO MENSAL NO BRASIL: EVIDÊNCIAS A PARTIR DE DADOS AGREGADOS DA PESQUISA FOCUS
}

\author{
Roberto Meurer * \\ Gilberto Tadeu Lima ${ }^{\dagger}$
}

\begin{abstract}
Resumo
Neste artigo é analisada a heterogeneidade das expectativas de inflação coletadas pelo Banco Central do Brasil, por meio de estatísticas descritivas e estimações econométricas para o comportamento da mediana, dispersão, amplitude e recorrência da presença de instituições no grupo Top 5, de maior acerto das previsões. São utilizadas expectativas agregadas do IPCA de janeiro de 2003 a agosto de 2016. Os resultados mostram correlação quase perfeita entre as previsões do conjunto de respondentes e os Top 5, ajuste gradual das expectativas, importância da data de referência para apuração do Top 5 e relação positiva entre variações da mediana e sua dispersão. O sistema de premiação das instituições Top 5 parece induzir uma parcela relevante dos respondentes a manter atualizadas as suas expectativas.
\end{abstract}

Palavras-chave: expectativas de inflação; heterogeneidade; Banco Central do Brasil.

\begin{abstract}
In this paper the heterogeneity of inflation expectations gathered by the Central Bank of Brazil is analyzed through descriptive statistics and econometric estimations for the median, dispersion, amplitude and recurrence of the presence of institutions in the Top 5 forecasters group. Aggregate expectations for the IPCA consumer price index from January 2003 to August 2016 are employed. Our results include an almost perfect correlation between the forecasts of all survey participants and the Top 5 forecasts, gradual adjustment of expectations, the significance of the reference day for the selection of the Top 5, and a positive relation between changes in the median and its dispersion. Rewarding the Top 5 seems to induce a relevant proportion of the survey participants to keep their forecasts updated.
\end{abstract}

Keywords: inflation expectations; heterogeneity; Brazilian Central Bank. JEL classification: E31, E37, E58

DOI: http : / dx.doi .org/10.11606/1980-5330/ea140932

\footnotetext{
* Universidade Federal de Santa Catarina, Departamento de Economia e Relações Internacionais. E-mail: roberto.meurer@ufsc.br

${ }^{\dagger}$ Universidade de São Paulo, Departamento de Economia/FEA
} 


\section{Introdução}

Quando a política monetária é conduzida em um sistema de metas para a inflação, a expectativa do mercado para a taxa de inflação e outras variáveis que influenciam a inflação é especialmente relevante (Svensson 1994). Em termos teóricos, há necessidade de se ter expectativas de inflação para caracterizar adequadamente a curva de Phillips, parte fundamental dos modelos macroeconômicos. No caso brasileiro, a implantação do sistema de metas para a inflação em 1999 levou à necessidade de coleta institucional sistemática das expectativas, o que passou a ser efetuado por meio da realização de pesquisa regular junto a participantes do mercado sobre o comportamento de diversas variáveis macroeconômicas.

As expectativas de inflação, tema deste artigo, são objeto de ampla literatura empírica (Coibion et al. 2017, Zarnowitz \& Braun 1993, Silveira \& Lima 2013, Guillén \& Garcia 2014). De outro lado, existe uma relação empírica e teórica estabelecida entre as expectativas de inflação e a credibilidade da política monetária (Bordo \& Siklos 2014, 2017, Lima et al. 2014).

Como as expectativas são um insumo importante para a condução da política monetária, o Banco Central do Brasil (BCB) criou uma competição entre os respondentes da sua pesquisa de expectativas que divulga mensalmente e premia anualmente os cinco respondentes com menor erro em suas previsões para taxa de juros Selic, taxa de câmbio e inflação. As previsões desse grupo, chamado de Top 5, para curto, médio e longo prazos, também são divulgadas separadamente.

Neste artigo são analisadas empiricamente relações entre as previsões referentes ao curto prazo, um mês, para a inflação medida pelo IPCA, considerando o agregado dos respondentes e o grupo Top 5. Com isso se enfatiza a formação das expectativas de inflação sem que estas sejam influenciadas por hipóteses específicas sobre a forma de condução da política monetária. Para tanto, são analisados, inclusive com estimativas econométricas, a mediana das previsões, seu desvio-padrão, sua amplitude e a recorrência da presença de instituições no grupo Top 5. O período de análise é de janeiro de 2003 a agosto de 2016, utilizando-se dados diários e mensais. ${ }^{1}$

$\mathrm{O}$ artigo apresenta uma breve revisão da metodologia da pesquisa de expectativas do BCB e da literatura sobre a pesquisa na seção 2 , a análise empírica na seção 3 e as principais conclusões na última seção. Na abordagem empírica, é efetuada análise descritiva (subseção 3.1), das variações na mediana das expectativas (subseção 3.2), da frequência de alterações na mediana (subseção 3.3) e da recorrência na participação das instituições no grupo Top 5 (subseção 3.4). Os resultados obtidos mostram que a mediana das expectativas do grupo Top 5 tem correlação muito forte com a mediana do conjunto de respondentes. Dos resultados, vale destacar também o ajuste gradual da mediana das expectativas e a relação positiva da variação da mediana com a dispersão das expectativas. Pelo menos uma parte importante dos respondentes da pesquisa do BCB ajusta as suas expectativas no mesmo dia ou no dia anterior à data de referência para apuração do grupo Top 5, o que foi evidenciado pela significância estatística das alterações de mediana das expectativas nesses dias.

\footnotetext{
${ }^{1}$ São utilizados os dados agregados divulgados pelo Banco Central do Brasil.
} 
Evidentemente não é possível, a partir dos dados utilizados, avaliar a efetividade ou eficácia da condução da política monetária no Brasil. Por outro lado, a detecção, mapeamento e quantificação de padrões de heterogeneidade na pesquisa de expectativas do BCB podem mostrar características de um importante insumo utilizado pelo $\mathrm{BCB}$ na condução da política monetária e pelos agentes da economia na construção e avaliação de cenários para a economia. Um melhor entendimento desses padrões, por sua vez, é um ingrediente importante para uma melhor compreensão e avaliação do comportamento da política monetária ao longo do período em questão.

\section{O sistema de expectativas do Banco Central do Brasil}

Existe uma longa tradição de coleta e divulgação de expectativas a partir de levantamentos com especialistas. O Livingstone Survey, para os Estados Unidos, é o mais antigo levantamento sistemático, iniciado em 1946 e atualmente sob responsabilidade do Philadelphia Federal Reserve. Dois dos principais são o Survey of Professional Forecasters (SPF) do Federeal Reserve (Fed) e o levantamento do Banco Central Europeu (ECB). O Survey of Professional Forecasters, atualmente realizado trimestralmente pelo Federal Reserve of Philadelphia, remonta a 1968 e cobre um amplo conjunto de variáveis macroeconômicas (Federal Reserve of Philadelphia 2016). O Banco Central Europeu também realiza trimestralmente um levantamento com previsores profissionais para inflação, PIB e desemprego na área do euro desde 1999 (European Central Bank 2016). Tanto o Fed quanto o ECB publicam as previsões individuais, não identificando o respondente.

No caso do Banco Central do Brasil (BCB), primeiramente foi criado um sistema de coleta direta de expectativas com participantes do mercado financeiro e consultorias, que evoluiu rapidamente para um sistema automatizado baseado na internet, a partir de 2001 (Federal Reserve of Philadelphia 2016).

A pesquisa de expectativas sobre variáveis macroeconômicas é efetuada pelo Banco Central do Brasil por meio do seu Departamento de Relacionamento com Investidores e Estudos Especiais (GERIN) e é conhecida como Pesquisa Focus. Os seus resultados são divulgados semanalmente no Focus - Relatório de Mercado e suas séries temporais estão disponíveis na internet por meio do Sistema de Expectativas de Mercado. São efetuados levantamentos para variáveis relacionadas a atividade econômica (PIB geral e setorial e produção industrial), meta da taxa de juros Selic, taxa de câmbio, preços (IPCA, IPCA-15, INPC, IPC-Fipe, IGP-DI, IGP-M, IPA-DI, IPA-M e preços administrados), balanço de pagamentos (balança comercial, conta corrente e investimento direto externo) e situação fiscal (resultado primário, resultado nominal e relação dívida/PIB). Para essas expectativas são divulgadas média, mediana, desvio-padrão, valores máximo e mínimo e coeficiente de variação. De acordo com o Banco Central do Brasil (2016), há cerca de 130 respondentes habilitados no sistema e boa parte altera suas previsões semanalmente.

As expectativas informadas são consolidadas diariamente pela GERIN do $\mathrm{BCB}$. As informações postadas no sistema têm validade de 30 dias e, caso não sejam atualizadas ou confirmadas, deixam de ser consideradas nas estatísticas das expectativas.

Como forma de incentivo para que as instituições respondentes gerem boas previsões o BCB divulga um ranking com as instituições com a maior 
acuidade das previsões, as Top 5. A classificação, com base no erro de previsão, é divulgada para IPCA, IGP-DI, IGP-M, taxa de câmbio e meta da taxa Selic. O ranking é divulgado para curto e médio prazos e para a previsão anual. Para a classificação de curto prazo é efetuado o cálculo do erro considerando a defasagem de um mês entre a previsão e o valor observado. O cálculo da média de erro é efetuado para um período de seis meses consecutivos. Para a classificação de médio prazo são considerados três períodos consecutivos de quatro meses, com as previsões sendo feitas ao longo desses quatro meses para o quarto mês. As previsões efetuadas com maior antecedência, ou seja, com menor disponibilidade de informações, têm maior peso no cálculo. Para o longo prazo é considerada a previsão ao longo dos 12 meses do ano para a variável observada ao final do ano, com pesos maiores para as previsões efetuadas no início do ano.

A metodologia de elaboração da classificação das instituições Top 5 pelo BCB define uma ou mais datas que são consideradas para a verificação do nível de acerto da previsão. A data de referência para o IPCA, objeto deste artigo, é o último dia útil anterior à data da divulgação do IPCA-15, em geral entre os dias 20 e 25.

Carvalho \& Minella (2012), usando dados desagregados, mostram que os melhores previsores influenciam as previsões dos demais respondentes na pesquisa do $\mathrm{BCB}$. Os respondentes reagem adaptativamente aos próprios erros e aos níveis passados das previsões. Um resultado importante é que as previsões são alinhadas entre os respondentes, sendo a maioria dos erros comuns, e não idiossincráticos. Para o caso específico da inflação, as previsões são similares ou melhores do que previsões baseadas em modelos autorregressivos. As previsões de inflação são influenciadas pela meta de inflação, inflação passada, taxa de câmbio e preço das commodities. Como esperado para um sistema de metas de inflação, o estudo mostra que as previsões para a taxa de juros da política econômica estão relacionadas com as expectativas de inflação. A similaridade das projeções de diferentes instituições também é encontrada em Caetano \& Silva Júnior (2012), também com dados desagregados, embora haja diferenças nas capacidades preditivas entre elas.

A forma como o ranking Top 5 é calculado faz com que haja inércia em sua composição. Os erros de previsão de um período são "carregados" para os períodos seguintes (Prada 2007). No caso do ranking de curto prazo, o erro em um período faz parte da média de erros por seis meses. No ranking de médio prazo o erro de um período terá impacto no máximo até o ranking de seis meses posteriores, com o peso atribuído à previsão mais longa. Para o ranking de longo prazo o erro da previsão de cada mês influencia o resultado com peso caindo a cada mês ao longo do ano.

Prada (2007) mostra, utilizando dados agregados de 2003 a 2006, que o nível de acerto da inflação medida pelo IPCA das instituições Top 5 é superior ao conjunto da pesquisa no período seguinte à entrada no grupo Top 5 , o que é indicativo de que as instituições que fazem parte desse grupo realmente são superiores em termos preditivos. O erro encontrado para os Top 5 é menor que para o total, e em $56 \%$ dos meses analisados a mediana das instituições Top 5 é mais próxima da inflação observada do que o conjunto de todas as previsões.

As projeções das instituições Top 5 não são sempre superiores às do conjunto de respondentes, o que mostra que a acurácia nas previsões passadas não assegura a continuidade da presença entre as instituições com as melho- 
res projeções (Marques 2012). Prada (2007) encontra, ao analisar a diferença entre as projeções de todos os respondentes da pesquisa e os Top 5 utilizando a teoria dos torneios, uma relação positiva entre o diferencial de desempenho e o desvio-padrão do total da população, indicando que quanto maior a dificuldade da previsão ou o risco corrido pelos previsores, maior a vantagem dos previsores mais hábeis. Uma maior dispersão das previsões significa uma maior dificuldade para efetuar as previsões ou a adoção de estratégias mais arriscadas por uma parcela dos previsores, o que aumenta a vantagem das instituições mais hábeis, que fazem previsões melhores e utilizam estratégias menos arriscadas (Prada 2007). Isso é evidenciado pela relação positiva entre o desvio-padrão das expectativas do conjunto das previsões e o diferencial de desempenho das instituições Top 5 e o conjunto. Outro resultado de Prada (2007) é a associação entre o diferencial de desempenho entre as instituições Top 5 e o esforço realizado para as previsões. Existindo heterogeneidade entre as instituições, as mais hábeis tenderão a aparecer mais vezes entre as Top 5. Entretanto, mesmo existindo heterogeneidade, o esforço dedicado às previsões pode variar. A medida de esforço utilizada é a permanência no grupo Top 5 por intervalos de dois a cinco meses e o número médio de permanências dessas instituições. O resultado estatisticamente significante encontrado é o coeficiente negativo para a permanência por cinco meses no ranking Top 5 sobre o diferencial de desempenho.

Rocha (2010) analisa as expectativas 12 meses à frente para IPCA, IPAM, IGP-M e IGP-DI coletadas entre novembro de 2001 e fevereiro de 2009, utilizando os dados agregados divulgados pelo BCB. A conclusão é que não há erro sistemático de previsão se excluído o período de 2002 a 2003 da amostra.

Um dos produtos do sistema de expectativas do BCB que é acompanhado com interesse no noticiário econômico e no mercado financeiro é o Boletim Focus divulgado às segundas feiras, mostrando as expectativas informadas na sexta feira anterior. Isso levanta o interessante ponto de que essas expectativas podem sofrer alterações relevantes, mesmo que não coincidam ou estejam próximas às datas de referência para a apuração do grupo Top 5. Existiriam, portanto, incentivos para que pelo menos parte importante das instituições respondentes da pesquisa respondessem fora da data de referência. Uma possível explicação seria a existência de demandas internas que tornam relevante ter uma expectativa atualizada, sendo o custo de atualização da expectativa no sistema do BCB baixo. Existe uma discussão na literatura sobre comportamento estratégico de respondentes de surveys, que poderiam ter incentivos para não informarem a sua real expectativa.

O modelo de Laster et al. (1999), por exemplo, associa a remuneração do previsor com a acurácia da previsão e com a publicidade gerada por ela. A publicidade é conseguida com previsões diferentes do consenso, o que induziria a informação de previsões enviesadas, mas com razoável probabilidade de ocorrência. Em sua aplicação a PIB e PNB dos Estados Unidos, a conclusão é de que as previsões informadas nos setores que dão muita importância à acurácia, como bancos e grandes corporações industriais, é mais próxima do consenso, enquanto setores que ganham mais com a publicidade gerada pela previsão tendem a produzir valores extremos. No caso do Top 5 do BCB , ao contrário do modelo de Laster et al. (1999), a publicidade é gerada pela acurácia, o que pode induzir à informação de expectativas que minimizem o erro esperado. Por outro lado, a maioria dos respondentes da pesquisa do BCB é do setor financeiro, corporações e consultorias, que tendem a ter interesse na 
publicidade gerada pela acurácia.

Lamont (2002) analisa a possibilidade de serem fornecidas previsões subótimas (automanipulation) quando a função de perda a ser minimizada pelo respondente não necessariamente é associada ao erro quadrático médio da previsão. A conclusão é que idade e reputação influenciam as previsões, com os previsores mais velhos e mais estabelecidos tendo previsões mais radicais. Essa intuição, entretanto, embora procedente, não é diretamente aplicável à pesquisa do $\mathrm{BCB}$ porque se refere a previsões fornecidas com identificação.

\section{Análise empírica}

\subsection{Análise descritiva}

A amostra das previsões é de 02/01/2003 a 31/08/2016, o que totaliza 164 meses e 3.429 dados diários. São utilizados os dados das medianas das previsões para o IPCA do mês efetuadas ao longo do mês, o seu desvio-padrão, a sua amplitude e a mediana e desvio-padrão das previsões do grupo Top 5. Para o grupo Top 5 o desvio-padrão está disponível a partir de 01/04/2010. Os dados são obtidos na página do Banco Central do Brasil. Como a expectativa utilizada é a do IPCA para o próprio mês, utiliza-se o Top 5 de curto prazo.

A divulgação do IPCA de um mês ocorre no início do mês seguinte. O horizonte da previsão, portanto, vai diminuindo ao longo do mês. A previsão utilizada pelo Banco Central do Brasil para a elaboração do ranking Top 5 é o dia anterior à divulgação do IPCA-15, o que em geral ocorre entre os dias 20 e 25. Esse dia é chamado de data de referência. O IPCA-15 é obtido com a mesma cesta de produtos do IPCA, mas em vez de refletir o comportamento dos preços no mês calendário, é calculado considerando os preços da segunda quinzena do mês anterior e a primeira do próprio mês.

Com essa amostra é possível calcular a variação da mediana das previsões para 3.265 dias, dado que não é possível comparar as previsões de inflação para meses distintos com o horizonte da previsão sendo de um mês. A alteração na mediana da expectativa ocorrerá se alterações nas previsões sensibilizarem a segunda casa decimal da previsão, ou seja, um centésimo de ponto percentual ou um ponto base. Para o conjunto dos respondentes ocorre alteração na mediana das previsões em 1.113 dias, o que corresponde a $34,1 \%$ dos dias. Para o grupo Top 5 a mediana é alterada em 773 dias, o que equivale a 23,7\% dos dias. Carvalho \& Minella (2012) informam que o conjunto de informantes regulares do sistema de expectativas do BCB é aproximadamente 70.

Para o conjunto dos respondentes a mediana é alterada em 119 das 164 datas de referência que ocorrem na amostra. As alterações de mediana das previsões na data de referência correspondem a 10,7\% do total de alterações, consideravelmente superior à proporção de datas de referência em relação ao número total de dias, que é de $5 \%$. A data de referência, portanto, está associada a uma maior atividade de alterações de previsão. Para o grupo Top 5 esse número é levemente superior, 123 alterações, que representa uma proporção de $15,9 \%$ das alterações na mediana.

A divulgação do IPCA-15 representa novas informações sobre o comportamento do IPCA do mês, sendo conhecido no noticiário como uma prévia do IPCA. Essas novas informações sobre a inflação podem ser incorporadas 
às previsões sobre o IPCA. Espera-se, por isso, que no dia da divulgação do IPCA-15 ocorra um maior ajuste nas previsões, embora em grau menor que na data de referência por essa previsão não ter impacto na elaboração do ranking Top 5. Ocorrem 62 alterações de mediana para o total de respondentes nesse dia, o que equivale a $5,6 \%$ do total de alterações, a 1,9\% do total de dias e $37,8 \%$ do total de meses. Para o grupo Top 5 ocorrem 42 alterações nesse dia, o que significa $5,4 \%$ do total de dias com alterações, $1,3 \%$ do total de dias e alteração em $25,6 \%$ dos meses. Esses números indicam que as novas informações que eventualmente a divulgação traz enseja um ajuste muito menor de previsões do que a data de referência. Esse resultado é indicativo de que o sistema de premiação Top 5 está induzindo a atualização das expectativas por parte dos respondentes, uma vez que as novas informações, que permitiriam uma expectativa mais apurada, são incorporadas com menor intensidade. Isso é confirmado pela não rejeição da hipótese nula de igualdade de média, mediana e variância dos erros de previsão para o IPCA na data de referência e na data de divulgação do IPCA-15, tanto para o conjunto de respondentes quanto para o grupo Top 5 (resultados não mostrados, mas disponibilizáveis aos leitores interessados).

Uma possibilidade adicional de ajuste informacional seria a que ocorre no dia seguinte à divulgação do IPCA-15. Poderia ocorrer um ajuste das previsões nesse dia se uma parcela dos respondentes tivesse como característica ser seguidor das previsões agregadas ou do grupo Top 5. As previsões efetuadas a cada dia da semana, entretanto, somente são disponibilizadas na segunda feira da semana-seguinte, tanto para o público quanto para os respondentes, o que não permite efetuar essa análise, a não ser que ocorresse uma troca de informações sobre as previsões fora do sistema do BCB. Ocorrem alterações em 42 desses dias no conjunto de todos os respondentes e em 18 do grupo Top 5. Por essas quantidades não há evidência de que esse mecanismo esteja operando de maneira expressiva o suficiente a ponto de ser detectado.

A Tabela 1 mostra os coeficientes de correlação entre a mediana agregada (MD), mediana Top 5 (MD5) e o desvio-padrão das previsões do conjunto de respondentes (DP). A Tabela 2 mostra os coeficientes de correlação incluindo o desvio-padrão para o grupo Top 5 (DP5), para um período mais restrito por causa da disponibilidade desse dado.

Tabela 1: Coeficientes de correlação entre mediana das expectativas de todos os respondentes e do grupo Top 5 - janeiro de 2003 a agosto de 2016

\begin{tabular}{lrcc}
\hline & MD & DP & MD5 \\
\hline MD & 1,000 & & \\
DP & 0,616 & 1,000 & \\
MD5 & 0,985 & 0,610 & 1,000 \\
\hline \multicolumn{4}{l}{ Fonte: Calculado a partir de } \\
dados do BCB.
\end{tabular}

Percebe-se uma correlação quase perfeita entre a mediana das previsões do agregado de respondentes e do grupo Top 5 para todo o período analisado e para o período mais recente, o que indica que esse grupo é uma boa aproximação do conjunto de respondentes. Há uma correlação positiva entre a dispersão das previsões do conjunto de respondentes e a mediana das previsões, 
Tabela 2: Coeficientes de correlação entre mediana das expectativas de todos os respondentes e do grupo Top 5 - abril de 2010 a agosto de 2016

\begin{tabular}{lrrrr}
\hline & MD & DP & MD5 & DP5 \\
\hline MD & 1,000 & & & \\
DP & 0,532 & 1,000 & & \\
MD5 & 0,985 & 0,516 & 1,000 & \\
DP5 & 0,208 & 0,622 & 0,206 & 1,000 \\
\hline
\end{tabular}

Fonte: Calculado a partir de dados do BCB.

ou seja, para uma mediana de previsão do IPCA mais elevada, a dispersão das previsões é maior. Considerando a hipótese de maior incerteza nas previsões estar associada com maior taxa de inflação (Ball 1992, Fountas 2001), esse resultado dá indicação de que a relação entre a incerteza nas previsões existe também para a própria taxa prevista de inflação. A influência do grupo Top 5 sobre as previsões de outros respondentes, encontrada por Carvalho \& Minella (2012) é apenas parcial, dado que a dispersão aumenta com o nível de inflação, o que não ocorreria se houvesse a simples imitação do grupo Top 5. Isso pode significar que a tendência à imitação cai à medida que sobe a taxa de inflação e/ou que a imitação é mais difícil e/ou menos bem sucedida com expectativas de taxas de inflação mais elevada.

Considerando o período mais recente, para o qual também existe a informação do desvio-padrão das expectativas do grupo Top 5, percebe-se uma redução da correlação entre a mediana das previsões e a sua dispersão. Por outro lado, a correlação entre a dispersão do grupo Top 5 e a mediana das suas previsões é baixa, o que mostra a proximidade das previsões dentro do grupo que mais acerta as previsões. Isso é interessante porque a previsão utilizada para a apuração do grupo Top 5 considera as previsões do período anterior, ou seja, o grupo que mais acertou no passado permanece tendo baixa divergência nas previsões para o período seguinte.

A existência de diferenças não desprezíveis na correlação entre a dispersão e o nível da inflação prevista quando calculado utilizando diferentes amostras sugere a necessidade de verificar em mais detalhe o comportamento dessa correlação. Para isso foi calculada a correlação entre a expectativa de inflação e a dispersão dessas expectativas utilizando janelas móveis de três anos. A Figura 1 mostra a correlação ao longo do tempo, considerando a data da informação mais recente presente no cálculo.

Esse comportamento das correlações é específico da previsão um mês à frente, em que a taxa de inflação já não é mais influenciada pela política monetária, e deve ser diferente para horizontes mais longos, em que a expectativa de inflação será influenciada pelas expectativas em relação à taxa de juros e outras variáveis que influenciam preços. Como se está utilizando previsões para a inflação mensal, o comportamento da correlação pode mudar rapidamente, com o comportamento específico de um determinado mês. No início da série de correlações, que contempla os dados de 2003 a 2005, por exemplo, a correlação é de 0,7 , que cai rapidamente quando incluídos os dados do primeiro semestre de 2006 e excluídos os do primeiro semestre de 2004 . A correlação cai consideravelmente porque nesse período mais recente quase não ocorrem alterações na dispersão das previsões, ao contrário do primeiro 
Figura 1: Correlação entre expectativa para o IPCA do mês e desviopadrão da expectativa - janelas móveis de três anos - janeiro de 2003 a agosto de 2016

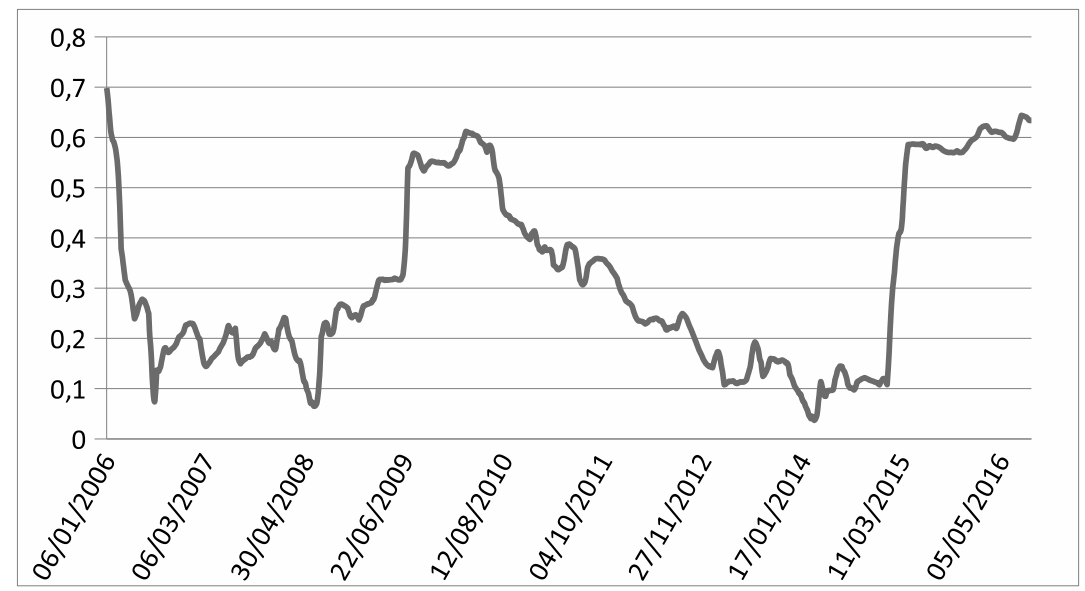

Fonte: Calculado a partir de dados do Banco Central do Brasil.

semestre de 2003, em que tanto a dispersão quanto a expectativa de inflação oscilaram fortemente.

O aumento da correlação que aparece em junho de 2009 deve-se à exclusão do mês de junho de 2006, em que a inflação esperada teve uma queda muito forte, saindo de 0,23 no início do mês para -0,1 no final do mês, com alterações muito menores na dispersão. A partir de julho de 2010 há um período de queda da correlação, caracterizado por poucas alterações no desvio-padrão das expectativas. A marcante elevação da correlação entre janeiro e março de 2015 ocorre em um cenário de elevação rápida da expectativa de inflação e na sua dispersão, refletindo o cenário econômico conturbado do período.

Esses resultados do comportamento das previsões para todos os respondentes e os Top 5 dão indicação de que estar no grupo Top 5 é relevante para pelo menos uma parcela importante dos respondentes. Isso pode ser ilustrado pelo comportamento da dispersão das previsões na data de referência para a previsão do IPCA. Considerando o conjunto de todos os respondentes, na data de referência ocorrem 75 reduções do desvio-padrão das expectativas, 15 elevações e em 74 dias não há alteração. Considerando todos os dias, ocorre redução do desvio-padrão em 492 dias, aumento em 312 e permanece inalterado em 2.461. Percebe-se, portanto, que a data de referência é caracterizada por um maior alinhamento de expectativas, com mais reduções de dispersão do que no conjunto da amostra. Para as previsões do grupo Top 5 o padrão é similar, com 14 elevações de dispersão, 48 reduções e 15 dias sem alteração, considerando os 77 meses para os quais existe a informação do desvio-padrão. Para todos os 1.539 dias para os quais existe o dado do desvio-padrão das previsões do grupo Top 5, existem 173 aumentos de dispersão, 208 reduções e 1.158 dias sem alteração.

Para o período em que também é divulgada a dispersão para o grupo Top 5, a partir de abril de 2010, a dispersão é consistentemente maior para o conjunto dos respondentes. Para os 1.616 dias desse período o desvio-padrão das previsões é maior no conjunto dos respondentes em 1.307 dias, maior para o grupo Top 5 em 175 e igual ao nível de centésimo de ponto percentual em 
134. Essa característica é compatível com um comportamento de menor risco e, por isso, de maior semelhança com o conjunto, por parte dos integrantes do grupo Top 5 ou os que competem mais proximamente para integrar esse grupo. Isso pode acontecer para previsores de instituições com departamentos econômicos grandes e qualificados, dos quais se espera que estejam entre os Top 5. Essa constatação é compatível com os resultados de Prada (2007), que associam esforço e habilidade a comportamentos menos arriscados e, com isso, a previsões mais próximas ao conjunto. Inversamente, previsores menos habilidosos teriam tendência a comportamentos mais arriscados. O resultado também está de acordo com o modelo de Laster et al. (1999), dados os incentivos que o sistema do BCB propicia para a informação de expectativas sem viés.

A amplitude das previsões, a diferença entre o maior e o menor valor informado ao sistema do BCB, permite ampliar a análise. O resultado das correlações para a amplitude, valor máximo e valor mínimo com as outras variáveis de interesse são mostradas na Tabela 3. Os valores máximo e mínimo para a expectativa de inflação têm correlação positiva e elevada com a mediana das expectativas, de 0,91 e 0,83 , respectivamente. A correlação entre o valor máximo e o mínimo é um pouco menor, de 0,69 . Interessante nesses resultados é que a correlação da amplitude das previsões é elevada com a expectativa máxima $(0,85)$ e baixa com o valor mínimo $(0,21)$. Isso pode indicar que os previsores pessimistas com a inflação ajustam mais as suas previsões, elevando o valor máximo em maior intensidade e reduzindo-o em menor intensidade que os agentes otimistas. De modo coerente, o desvio-padrão das expectativas tem uma elevada correlação de 0,93 com a amplitude das previsões. Novamente, a origem dessa correlação elevada é mais influenciada pelo comportamento do valor máximo, pois a correlação entre dispersão e valor máximo é elevada, 0,8 , enquanto entre dispersão e valor mínimo é baixa. Esses resultados confirmam as indicações de que elevação da inflação prevista é acompanhada de aumento da dispersão, tanto medida pelo desvio-padrão quanto pela amplitude das previsões.

Tabela 3: Coeficientes de correlação - expectativas para o IPCA do mês janeiro de 2003 a agosto de 2016 - dados diários

\begin{tabular}{lccccc}
\hline & Amplitude & Máximo & Mínimo & Mediana & Desvio Padrão \\
\hline Amplitude & 1,000 & & & & \\
Máximo & 0,847 & 1,000 & & & \\
Mínimo & 0,205 & 0,693 & 1,000 & & \\
Mediana & 0,624 & 0,912 & 0,835 & 1,000 & \\
Desvio Padrão & 0,928 & 0,797 & 0,209 & 0,616 & 1,000 \\
\hline
\end{tabular}

Fonte: Calculado a partir de dados do BCB.

A importância da obtenção de expectativas confiáveis, como as incentivadas pela divulgação das instituições Top 5, pode ser ilustrada pela elevada amplitude das expectativas de inflação quando comparadas à sua mediana, como mostra a Figura 2. O valor absoluto da amplitude das expectativas é elevado comparativamente à mediana, o que torna relevante que esta reflita as melhores previsões. Por outro lado, as medidas de dispersão como o desviopadrão e a própria amplitude podem conter informação sobre a coordenação das expectativas entre os participantes da pesquisa. Isso é sugestivamente dis- 
cutido em Mankiw, Mankiw et al. (2003), que mostram que a discordância sobre as expectativas em relação à inflação está relacionada com discordância em relação a outras variáveis macroeconômicas importantes. Como as decisões individuais são baseadas em expectativas, se houver discordância entre as expectativas, isso poderá afetar o resultado agregado e, por isso, a condução da política monetária.

Figura 2: Mediana e amplitude das previsões de inflação - janeiro de 2003 a agosto de 2016

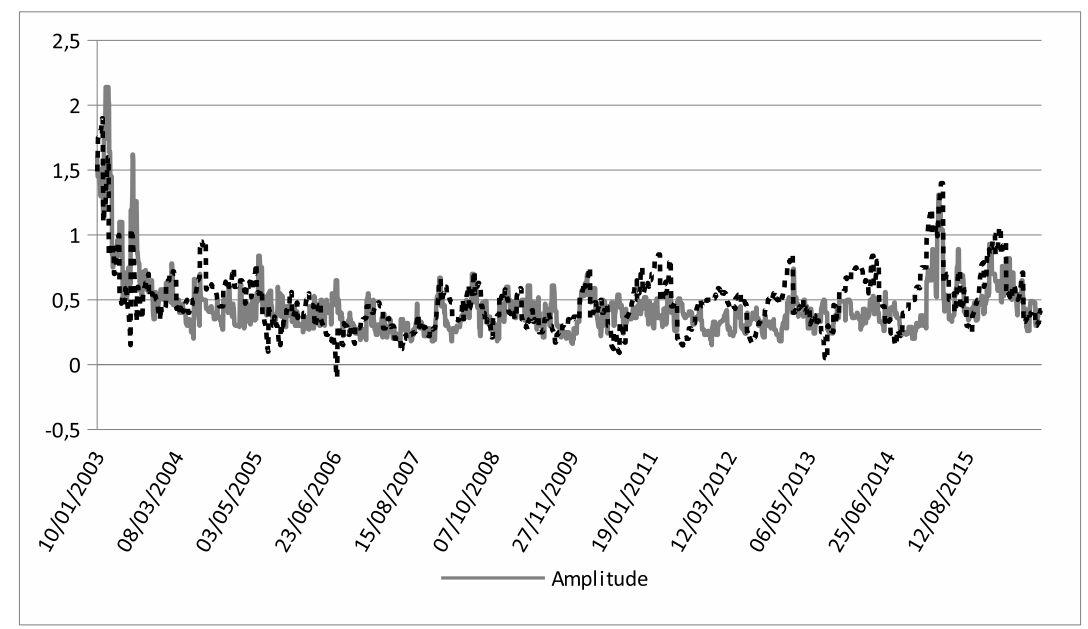

Fonte: Mediana - BCB; Amplitude - calculada a partir de dados do BCB

\subsection{Variações na mediana das expectativas}

Para quantificar o comportamento das previsões, foram estimadas regressões tendo como variável dependente a variação absoluta da mediana das expectativas de inflação para todos os respondentes (VMDABS) e para o grupo Top 5 (VMD5ABS). Foi utilizada a variação da expectativa e não o seu nível por não ser possível comparar os níveis da inflação para meses diferentes, dadas as características específicas de cada mês. Com as variações, os aspectos comuns a alterações de expectativas informadas em todo o período podem ser detectados. Utilizaram-se as variações absolutas das expectativas porque as variações para cima ou para baixo também estão sujeitas a condicionantes econômicas específicas de cada período, de modo que ajustes para cima ou para baixo nas expectativas poderiam se compensar ao longo do tempo ou simplesmente seguir a tendência da inflação. As estimativas foram efetuadas partindo de um modelo geral contendo duas defasagens para a variação das expectativas, a mediana da expectativa, o desvio-padrão das expectativas de todos os respondentes (DP) e variáveis dummy que assumem valor 1 na data de referência e zero nos demais dias (DATAREF), bem como a mesma variável dummy para um e dois dias antes da data de referência e um e dois dias após a data de referência (DATAREF(-1), DATAREF(-2) DATAREF(1) DATAREF(2), respectivamente). As possibilidades de assimetria de comportamento da mediana das expectativas para situações de elevação ou queda da inflação também foram testadas. Uma dummy assumiu valor 1 se a variação da mediana fosse positiva e zero caso contrário, a outra assumiu valor 1 se a variação da mediana fosse 
negativa e zero caso contrário. Essas dummies, entretanto, não apresentaram significância estatística.

Seguindo o procedimento geral-específico e eliminando as variáveis não estatisticamente significantes, chegou-se ao modelo parcimonioso mostrado na Tabela 4. Foram utilizados os erros-padrão robustos à heteroscedasticidade de Newey-West.

Tabela 4: Resultados da regressão - variável dependente: VMDABS - 01/06/2003 a 30/08/2016

\begin{tabular}{lrcrc}
\hline \multicolumn{1}{c}{ Variável } & Coeficiente & Desvio-Padrão & Estatística $\mathbf{t}$ & Prob. \\
\hline VMDABS(-1) & 0,087772 & 0,026162 & 3,354932 & 0,0008 \\
DATAREF & 0,017918 & 0,001694 & 10,577880 & 0,0000 \\
DATAREF(1) & 0,003347 & 0,000945 & 3,543205 & 0,0004 \\
MD & $-0,009333$ & 0,001412 & $-6,611256$ & 0,0000 \\
DP & 0,117827 & 0,011018 & 10,693870 & 0,0000 \\
\hline
\end{tabular}

$R^{2}: 0,257 ; R^{2}$ ajustado: 0,2563 .

A segunda defasagem da variação da mediana e as dummies para os dois dias anteriores e o segundo dia após a data de referência não foram estatisticamente significativos. O termo autorregressivo de primeira ordem é positivo, o que é plausível considerando que a mediana é resultante das previsões de muitos agentes que não atualizam as suas previsões exatamente ao mesmo tempo, ou seja, há um ajuste gradual e escalonado das expectativas para o conjunto dos respondentes. As previsões podem ter esse comportamento autorregressivo por estarem reagindo a um mesmo conjunto de informações, mesmo que não concomitantemente, ou seguindo um comportamento imitativo entre os próprios respondentes, ou uma combinação entre elas. Se utilizada a variação da mediana defasada do grupo Top $5 \mathrm{em}$ vez da do conjunto de respondentes os resultados são muito parecidos, com poder explicativo marginalmente inferior $\left(R^{2}\right.$ de 0,2543 contra 0,2563$)$. Como esperado, há um ajuste estatisticamente significativo nas expectativas na data de referência, o que novamente indica que participar do grupo Top 5 é importante para um número relevante de respondentes da pesquisa. A hipótese de que a divulgação do IPCA-15 traz informações para o comportamento do IPCA do mês e é incorporada às expectativas é confirmada pela significância da dummy para o dia da divulgação do IPCA-15. O valor do coeficiente é menor do que o da data de referência. A dummy para o dia seguinte à divulgação do IPCA-15 não foi estatisticamente significante, o que não dá suporte à hipótese de que pelo menos parte dos respondentes da pesquisa ajusta as expectativas por imitação de expectativas, $o$ que é esperado dado que as expectativas diárias somente são disponibilizadas às segundas feiras no sistema do $\mathrm{BCB}$, ou de incorporação lenta das informações trazidas pelo IPCA-15.

O desvio-padrão das expectativas tem relação positiva com as variações absolutas da mediana, ou seja, quanto maior a dispersão das expectativas, maiores os ajustes nas expectativas. O mais plausível parece ser que os ajustes das expectativas não são feitos na mesma intensidade e sincronização por todos os respondentes, levando a uma maior dispersão das expectativas. Isso poderia ocorrer por causa da estrutura de incentivos inerente à pesquisa e sua premiação por acurácia nas datas de referência. Do ponto de vista teórico, esse comportamento é compatível, por exemplo, com a explicação presente em Branch 
(2004), de que a heterogeneidade das previsões decorre da existência de alternativas para a realização das previsões, as quais têm custos diferentes para sua utilização.

O resultado negativo para a relação entre o nível da mediana e a variação da mediana das expectativas pode indicar a necessidade de ajustes mais frequentes nas expectativas quando a inflação é mais elevada, mas em valor absoluto menor. O impacto de erros de previsão da inflação sobre as decisões econômicas é maior quando o nível da inflação é mais elevado, exigindo dessa forma atenção mais cuidadosa e alterações de previsões mais frequentes quando a inflação é elevada em comparação com inflações esperadas menores. Esse resultado é compatível com a persistência nas variações da mediana detectada por meio do valor positivo da sua autocorrelação.

As Tabelas 5 e 6 mostram os resultados da regressão que tem a variação da mediana da expectativa de inflação do grupo Top 5 como variável dependente. A diferença entre as duas é a utilização da dispersão do conjunto de respondentes na regressão da Tabela 5 e da dispersão das previsões do grupo Top 5 na Tabela 6. A amostra utilizada para a estimativa mostrada na Tabela 6 é menor, dada a disponibilidade da informação sobre o desvio-padrão das expectativas do grupo Top 5 para um período mais restrito, a partir de abril de 2010.

Tabela 5: Resultados da regressão - variável dependente: VMD5ABS $-01 / 06 / 2003$ a $30 / 08 / 2016$

\begin{tabular}{lrcrc}
\hline \multicolumn{1}{c}{ Variável } & Coeficiente & Desvio-Padrão & Estatística t & Prob. \\
\hline VMD5ABS(-2) & $-0,076778$ & 0,021625 & $-3,550390$ & 0,0004 \\
DATAREF & 0,025682 & 0,002731 & 9,403055 & 0,0000 \\
DATAREF(1) & 0,012934 & 0,003009 & 4,298613 & 0,0000 \\
MD5 & $-0,007331$ & 0,002978 & $-2,462033$ & 0,0139 \\
DP & 0,136847 & 0,022571 & 6,063079 & 0,0000 \\
\hline
\end{tabular}

$R^{2}: 0,119 ; R^{2}$ ajustado: 0,118 .

Tabela 6: Resultados da regressão - variável dependente: VMD5ABS - 05/04/2010 a 30/08/2016

\begin{tabular}{lrcrc}
\hline \multicolumn{1}{c}{ Variável } & Coeficiente & Desvio-Padrão & Estatística $\mathbf{t}$ & Prob. \\
\hline DATAREF $(-2)$ & $-0,002219$ & 0,000884 & $-2,509791$ & 0,0122 \\
DATAREF & 0,032191 & 0,004166 & 7,727280 & 0,0000 \\
DATAREF $(1)$ & 0,012430 & 0,003571 & 3,480695 & 0,0005 \\
DP5 & 0,104455 & 0,009712 & 10,755040 & 0,0000 \\
\hline$R^{2}: 0,171 ; R^{2}$ ajustado: $0,170$. & & &
\end{tabular}

Os resultados obtidos para o período mais longo apresentam semelhanças importantes em comparação ao conjunto de respondentes. A data de referência e a divulgação do IPCA-15 também têm efeitos positivos sobre a variação da mediana das expectativas, em magnitudes maiores do que para o conjunto dos respondentes. Como o grupo Top 5 é numericamente mais restrito e já demonstrou sua capacidade preditiva, é razoável esperar que na data de referência para a elaboração do ranking haja uma maior variação nas suas expectativas, bem como um maior ajuste das expectativas em reação à divulgação de 
novas informações sobre o comportamento dos preços. Por outro lado, isso demonstra que não é somente a inclusão no ranking Top 5 que estimula a revisão das expectativas, uma vez que ocorrem ajustes relevantes no dia da divulgação das informações, embora essas previsões não tenham influência no ranking. A mediana das previsões, ou seja, o nível das expectativas de inflação, tal como para o conjunto dos respondentes, tem relação negativa com a variação das expectativas, assim como relação positiva com a dispersão das expectativas. Tal como no resultado para o conjunto dos respondentes, esse resultado pode refletir a necessidade de maior quantidade de ajustes quando a expectativa de inflação é mais elevada, mas a magnitude da alteração de expectativa ser menor. O comportamento dos respondentes que gera esses resultados pode refletir, por exemplo, os custos e necessidades de atualização de previsões para a demanda da própria instituição e a sua atualização no sistema de expectativas do BCB, e não caracteriza um comportamento que considera somente o desempenho na data crítica para o levantamento Top 5.

Uma característica consideravelmente distinta no comportamento da variação das expectativas se dá no efeito das variações dos dias anteriores. Enquanto para o conjunto o coeficiente autorregressivo é positivo, para o grupo Top 5 o coeficiente é negativo para duas defasagens. Isso poderia indicar que existe algum custo para a atualização ou o registro das expectativas para esse grupo de respondentes da pesquisa, a ponto de ajustes maiores em dias anteriores estarem associados a menores ajustes correntes. Outra possibilidade é que a diferença de comportamento reflita um padrão distinto para esse grupo de respondentes, mesmo que a sua composição não seja sempre a mesma, possivelmente refletindo a sua capacidade preditiva.

O resultado para o período mais recente das variações absolutas da mediana da expectativa de inflação do grupo Top 5, utilizando a dispersão das expectativas do próprio grupo Top 5 entre as variáveis explicativas é similar ao do período integral. As dummies para a data de referência e a divulgação do IPCA-15 permanecem significativas. A relação entre a dispersão das expectativas e a variação da mediana também permanece positiva. Por outro lado, o nível da mediana de inflação esperada deixa de ser significativa. Isso pode estar relacionado a uma mudança de comportamento dos previsores ao longo do tempo ou à diferença entre o modelo utilizando a dispersão do conjunto de respondentes e do grupo Top 5. Essa mudança de comportamento pode refletir, por exemplo, alterações na maneira como é feita a previsão ou mudanças na própria inflação. Isso poderia ocorrer, por exemplo, com a observação das previsões do grupo Top 5 e adaptação ao desempenho que se mostrou bem sucedido. Também ocorre uma mudança na estrutura temporal das variações, com as variações na mediana de períodos anteriores não tendo significância estatística e a dummy para dois dias antes da data de referência passando a ser estatisticamente significante e o coeficiente negativo indicando que as variações nessa data são menores que nos demais dias. Isso significa que dois dias antes da data de referência o ajuste que é efetuado nas previsões registradas no sistema do Banco Central é significativamente menor. Isso pode indicar que dois dias antes da data de referência os participantes do grupo Top 5 deixam de fazer ajustes ou o fazem de maneira muito marginal, aguardando para incorporar o máximo de informação possível para a previsão na data de referência. Alternativamente, pode-se conjecturar que, como esse grupo é o dos melhores preditores, necessita de ajustes menores na data de referência Como as maiores variações de expectativas são registradas na data de referência, os 
modelos das Tabelas 5 e 6 detectam comportamentos similares, embora a interpretação da dummy para o dia específico seja mais restrita do que a defasagem da variação.

\subsection{Frequência de alterações na mediana}

Para confirmar as diferenças de comportamento entre as instituições Top 5 e o conjunto de respondentes foi efetuado o levantamento do número de alterações na mediana das previsões que ocorre em cada mês da amostra e buscou-se verificar as variáveis que influenciam a quantidade de alterações. Uma alteração na mediana das previsões ocorre se um número suficiente de instituições alterar a sua projeção em magnitude suficiente para sensibilizar a segunda casa decimal da taxa de inflação prevista. Isso significa que tem de haver alguma predominância no sentido da alteração das previsões, porque alterações individuais em sentidos contrários sem grandes diferenças de magnitude poderiam se anular. A Tabela 7 sintetiza o número de alterações na mediana ocorridas no período. Como esperado, tendo um número reduzido de respondentes, as alterações no grupo Top 5 ocorrem em número inferior, bem como a moda do número de alterações, mas é importante lembrar que a importância relativa de um respondente no grupo Top 5 é maior do que no conjunto de respondentes para que ocorra mudança na mediana das previsões.

A ocorrência mais comum no conjunto de respondentes é de cinco e seis alterações na mediana ao longo do mês, que ocorre em 22 e 23 meses, respectivamente. Esses números correspondem a 13,5 e 14,1 do total de 163 meses que compõem a amostra. Em somente 31 meses ocorrem 10 ou mais alterações na mediana, menos de $20 \%$ do total de meses da amostra. Para o grupo top 5 o número de alterações na mediana que mais ocorre é 3, em 33 meses, e o segundo mais frequente é cinco alterações, em 31 meses, o que corresponde a $20 \%$ e $19 \%$ do total de 163 meses da amostra. A ocorrência de sete ou mais alterações na mediana para esse grupo ocorre em menos de $10 \%$ dos meses.

Para a análise econométrica da frequência de alterações na mediana (NALT) é necessário considerar que esta é uma variável discreta, assumindo necessariamente valores inteiros iguais ou maiores que zero. Por isso é necessário utilizar modelos de contagem para a análise desses dados. O ponto de partida natural para essa abordagem é a estimação de modelos Poisson que, entretanto, são restritivos quanto aos pressupostos em relação ao comportamento de média e variância, que são considerados iguais. É necessário, portanto, primeiramente testar a hipótese de igualdade entre média e variância condicional às variáveis explicativas que, se rejeitada, implica a necessidade de estimar modelos alternativos. Para isso será utilizado o teste baseado em regressão proposto por Cameron \& Trivedi (1990).

Utilizou-se a metodologia geral-específico mantendo as variáveis significativas a no mínimo $10 \%$. O modelo mais amplo incluiu como variáveis explicativas a inflação acumulada nos 12 meses anteriores, o IPCA do mês anterior (IPCA(-1)), o erro da previsão ocorrido no mês anterior (ERROABS(-1)) e a volatilidade das expectativas ao longo do mês (DPTODOS) e do mês anterior (DPTODOS(-1)). Também foi considerada a hipótese de utilizar os desvios da inflação em relação à meta, mas a correlação quase perfeita entre os desvios da meta e da inflação acumulada não permitem a inclusão das duas variáveis simultaneamente nos modelos estimados. A elevada correlação entre inflação e desvio da inflação em relação à meta decorre de as alterações na meta, no pe- 
Tabela 7: Número de alterações na mediana das expectativas ao longo de cada mês - janeiro de 2003 a agosto de 2016

\begin{tabular}{|c|c|c|c|c|}
\hline \multicolumn{5}{|c|}{ CONJUNTO DE RESPONDENTES } \\
\hline $\begin{array}{l}\text { Número de } \\
\text { alterações na } \\
\text { mediana no } \\
\text { mês }\end{array}$ & $\begin{array}{l}\text { Quantidade } \\
\text { de meses em } \\
\text { que ocorreu } \\
\text { esse número } \\
\text { de alterações } \\
\text { no período }\end{array}$ & $\begin{array}{l}\text { Percentual do } \\
\text { total de meses }\end{array}$ & $\begin{array}{c}\text { Número } \\
\text { de meses } \\
\text { acumulado }\end{array}$ & $\begin{array}{l}\text { Percentual } \\
\text { acumulado }\end{array}$ \\
\hline 0 & 4 & 2,5 & 4 & 2,5 \\
\hline 1 & 4 & 2,5 & 8 & 4,9 \\
\hline 2 & 4 & 2,5 & 12 & 7,4 \\
\hline 3 & 14 & 8,6 & 26 & 16,0 \\
\hline 4 & 11 & 6,7 & 37 & 22,7 \\
\hline 5 & 22 & 13,5 & 59 & 36,2 \\
\hline 6 & 23 & 14,1 & 82 & 50,3 \\
\hline 7 & 16 & 9,8 & 98 & 60,1 \\
\hline 8 & 17 & 10,4 & 115 & 70,6 \\
\hline 9 & 17 & 10,4 & 132 & 81,0 \\
\hline 10 & 10 & 6,1 & 142 & 87,1 \\
\hline 11 & 9 & 5,5 & 151 & 92,6 \\
\hline 12 & 4 & 2,5 & 155 & 95,1 \\
\hline 13 & 3 & 1,8 & 158 & 96,9 \\
\hline 14 & 4 & 2,5 & 162 & 99,4 \\
\hline 15 & 1 & 0,6 & 163 & 100,0 \\
\hline \multicolumn{5}{|c|}{ TOP 5} \\
\hline 0 & 1 & 0,6 & 1 & 0,6 \\
\hline 1 & 5 & 3,1 & 6 & 3,7 \\
\hline 2 & 15 & 9,2 & 21 & 12,9 \\
\hline 3 & 33 & 20,2 & 54 & 33,1 \\
\hline 4 & 24 & 14,7 & 78 & 47,9 \\
\hline 5 & 31 & 19,0 & 109 & 66,9 \\
\hline 6 & 25 & 15,3 & 134 & 82,2 \\
\hline 7 & 14 & 8,6 & 148 & 90,8 \\
\hline 8 & 8 & 4,9 & 156 & 95,7 \\
\hline 9 & 4 & 2,5 & 160 & 98,2 \\
\hline 10 & 1 & 0,6 & 161 & 98,8 \\
\hline 11 & 1 & 0,6 & 162 & 99,4 \\
\hline 12 & 1 & 0,6 & 163 & 100,0 \\
\hline
\end{tabular}


ríodo analisado, terem sido pequenas, passando de $4 \%$ para $2003,5,5 \%$ para 2004 e permanecendo em 4,5\% a partir de 2005. A inflação passada foi testada utilizando o índice cheio e diferentes medidas de núcleo da inflação. $\mathrm{O}$ erro da expectativa de inflação no mês anterior considerou a previsão registrada no primeiro dia do mês para o qual foi feita a previsão. A volatilidade das expectativas é o desvio-padrão da mediana das expectativas ao longo do mês. As variáveis de inflação acumulada em 12 meses e erro de previsão no período anterior não foram estatisticamente significantes.

O resultado da estimação é mostrado na Tabela 8. Em se tratando de um modelo de contagem que é uma série temporal, foi verificada a ausência de autocorrelação dos resíduos. A dispersão das previsões do conjunto de respondentes no primeiro dia do próprio mês tem uma forte relação positiva com o número de alterações da mediana. Esse resultado é intuitivo porque a maior dispersão das expectativas se reflete em um maior número de ajustes, uma vez que a informação a ser incorporada nas previsões é incremental ao longo do mês. O nível da inflação no mês anterior tem relação negativa com o número de alterações da mediana da previsão no mês. Uma tentativa de utilizar as medidas de núcleo da inflação divulgadas pelo BCB em lugar da inflação observada piorou o ajuste do modelo. O intercepto da estimativa $(\operatorname{EXP}(1,652166)=5,218)$ não tem uma interpretação direta, porque significaria o número de alterações na mediana caso o IPCA do período anterior e o desvio-padrão das expectativas fosse zero, o que não é realista. A magnitude do coeficiente, entretanto, mostra que as variáveis explicativas têm influência considerável na determinação do número de alterações na mediana, cujo valor médio é de 6,78 no período considerado.

Tabela 8: Resultado da regressão - variável dependente: NALT fevereiro de 2003 a agosto de 2016 - modelo de contagem - Poisson

\begin{tabular}{lcccc}
\hline Variável & Coeficiente & Desvio-Padrão & Estatística z & Prob. \\
\hline Constante & 1,652166 & 0,070353 & 23,483910 & 0,0000 \\
IPCA(-1) & $-0,189654$ & 0,102056 & $-1,858329$ & 0,0631 \\
DPTODOS & 4,113006 & 0,779216 & 5,278388 & 0,0000 \\
\hline
\end{tabular}

$R^{2}$ : 0,107; $R^{2}$ ajustado: 0,095; Erro-padrão da regressão: 3,038.

O coeficiente estimado no modelo de contagem pode ser interpretado como a mudança relativa esperada na variável dependente induzida por uma alteração de uma unidade na variável explicativa. A mudança na variável dependente, portanto, depende do seu nível. Para o resultado apresentado na Tabela 8 , um aumento de um ponto percentual na inflação de um período estaria associado a uma redução de $19 \%$ no número de alterações na mediana das previsões no período seguinte. Uma possível explicação para esse resultado está no custo mais elevado associado a erros de previsão com inflação mais alta, o que levaria a um maior esforço e dilegência na elaboração das previsões no período seguinte, algo que possivelmente demandaria, por consequência, menor número de revisões dessas mesmas expectativas. De modo similar, uma elevação de 0,1 ponto percentual no desvio-padrão das expectativas em um mês estaria associado a um número $41 \%$ maior de alterações na mediana no próprio mês. 
Foi estimado um modelo alternativo utilizando o erro de previsão do grupo Top 5 (ERROABS5(-1)) em lugar do erro de previsão do conjunto dos respondentes, cujo resultado final é apresentado na Tabela 9. Os resultados são de um modelo binomial negativo, uma vez que a adequação do modelo Poisson foi rejeitada. A principal diferença nos resultados é a relação positiva entre o erro de previsão no mês anterior com o número de alterações na mediana no mês corrente. Esse resultado indica que há algum efeito das projeções e erros do grupo Top 5 sobre o conjunto de respondentes.

Tabela 9: Resultado da regressão - variável dependente: NALT - fevereiro de 2003 a agosto de 2016 - modelo de contagem - binomial negativa

\begin{tabular}{lcccc}
\hline Variável & Coeficiente & Desvio-Padrão & Estatística $\mathrm{z}$ & Prob. \\
\hline Constante & 1,597316 & 0,082589 & 19,340530 & 0,0000 \\
DPTODOS & 2,669740 & 0,942232 & 2,833421 & 0,0046 \\
ERROABS5(-1) & 0,534691 & 0,303942 & 1,759186 & 0,0785 \\
\hline$R^{2}: 0,112 ; R^{2}$ ajustado: 0,$101 ;$ Erro-padrão da regressão: & 3,028.
\end{tabular}

A Tabela 10 mostra os resultados da regressão do número de alterações que ocorre na mediana das previsões no grupo Top 5 (NALT5). Há diferenças relevantes em relação aos resultados para o conjunto de todos os respondentes. O teste para correlação serial dos resíduos mostrou a existência de correlação, motivo pelo qual foi incluído um termo autorregressivo na estimação. O teste de Cameron e Trivedi para a regressão incluindo todas as variáveis explicativas mostrou a adequação do modelo Poisson, assim como para suas sucessivas reduções. O resultado final, mantendo somente as variáveis estatisticamente significantes, apresenta somente a constante e o termo autorregressivo. A interpretação desse resultado é que o número de alterações que ocorre na mediana das previsões do grupo Top 5 tem uma inércia, dada pelo termo autorregressivo, mas não reage sistematicamente ao conjunto de variáveis utilizado. Isso pode ser indicativo de que as alterações na mediana ocorrem concomitantemente à geração de novas informações que podem influenciar a inflação, sem que haja tempo de serem captadas por medidas cuja mensuração ocorre em intervalos mensais. Em outras palavras, parece haver eficiência na geração das previsões do grupo Top 5, que altera as previsões rapidamente com a percepção dessa mudança.

Tabela 10: Resultado da regressão - variável dependente: NALT5 - Fevereiro de 2003 a agosto de 2016

\begin{tabular}{lcccc}
\hline Variável & Coeficiente & Desvio-Padrão & Estatística z & Prob. \\
\hline C & 1,274926 & 0,090641 & 14,065600 & 0,0000 \\
NALT5(-1) & 0,056332 & 0,016739 & 3,365218 & 0,0008 \\
\hline$R^{2}: 0,077 ; R^{2}$ ajustado: 0,071; Erro-padrão da regressão: $2,015430$. &
\end{tabular}

\subsection{Recorrência}

A análise de recorrência visa avaliar quais são as variáveis associadas a um índice de recorrência (INDREC), que mede a permanência das instituições que 
estão no grupo Top 5 nesse grupo no período seguinte. Para isso foi montado um índice de recorrência, calculando a proporção do número de instituições do grupo Top 5 em um período que também estavam nesse grupo no período imediatamente anterior. Por construção, essa proporção estará entre zero e um. Para verificar a existência de explicações para a permanência maior ou menor das instituições no grupo Top 5, foi estimada a regressão tendo como variável dependente o índice de recorrência e como variáveis explicativas os valores do mês e do mês anterior da taxa do IPCA, IPCA acumulado em 12 meses, erro de previsão do conjunto de respondentes e do grupo Top 5, média do desvio-padrão das expectativas ao longo do mês para o conjunto de respondentes e o grupo Top 5, desvio-padrão das expectativas no primeiro dia do mês do conjunto de respondentes e do grupo Top 5. Somente o erro absoluto da previsão e a média do desvio-padrão das previsões do grupo Top 5 ao longo do mês foram estatisticamente significantes, como pode ser observado na Tabela 11.

Tabela 11: Resultado da regressão - variável dependente: INDREC - abril de 2003 a agosto de $2016-\mathrm{N}=161$

\begin{tabular}{lrcrc}
\hline Variável & Coeficiente & Desvio-Padrão & Estatística t & Prob. \\
\hline Constante & 0,610513 & 0,025361 & 24,073370 & 0,0000 \\
ERROABS & $-0,504530$ & 0,200398 & $-2,517645$ & 0,0128 \\
DP5MES & 1,421984 & 0,640460 & 2,220254 & 0,0278 \\
\hline$R^{2}$ & 0,039391 & & & \\
$R^{2}$ ajustado & 0,027232 & & & \\
F-statistic & 3,239533 & Prob. & 0,0418 & \\
$\begin{array}{l}\text { Breusch-Godfrey Serial } \\
\text { Correlation LM Test }\end{array}$ & 0,734797 & Prob. & 0,4813 & \\
Heteroskedasticity Test: & 1,077524 & Prob. & 0,3429 & \\
$\begin{array}{l}\text { Breusch-Pagan-Godfrey } \\
\text { Ramsey RESET Test: }\end{array}$ & 0,137959 & Prob. F(1,157) & 0,7108 & \\
\hline
\end{tabular}

A constante estimada é de valor praticamente idêntico à média da amostra, que é de 0,6. O erro absoluto da previsão, medida pela diferença entre a inflação observada e a mediana da previsão realizada no primeiro dia do mês, tem efeito negativo sobre o índice de recorrência. Isso é plausível, considerando que um erro maior está relacionado com maior dificuldade de acertar a previsão em função de alterações de cenário ao longo do mês, indicando que mesmo para as instituições com maior habilidade parece haver maior dificuldade em permanecer no grupo Top 5 quando há maiores mudanças no cenário para a inflação do mês. Essa maior dificuldade afeta a maior parte das instituições, inclusive aquelas que estavam no grupo Top 5. Isso poderia indicar, também, que o acerto ocorreu de maneira aleatória, possivelmente refletindo uma estratégia mais arriscada, mesmo que sejam as instituições mais hábeis as que tendam a estar no grupo Top 5 (Prada 2007). Outra maneira de ver esse resultado seria que um maior erro pode tornar o acerto mais aleatório e, por isso, quais instituições mais se aproximam do dado observado também poderia se tornar uma ocorrência mais aleatória. Dada a proximidade da mediana da expectativa do grupo Top 5 e do conjunto de respondentes, a regressão estimada com o erro do grupo Top 5 gera praticamente o mesmo resultado, com 
coeficiente de -0,519 e erro-padrão de 0,2.

O resultado para a dispersão da mediana das expectativas do próprio grupo Top 5 mostra que uma maior dispersão das expectativas ao longo do mês está associada a uma maior recorrência no grupo Top 5. Uma possível interpretação para esse resultado é que, quando a dispersão é maior, as instituições Top 5 se destacam mais em sua capacidade preditiva. Isso significa uma habilidade por parte dessas instituições em permanecerem no grupo Top 5 quando há uma maior dispersão das expectativas. É interessante que, quando estimada a regressão com a dispersão para o conjunto dos respondentes, esta não foi estatisticamente significante. Isso indica que a maior dispersão nas previsões efetuadas entre as instituições que estão no grupo Top 5 é que está associada à maior possibilidade de permanência no grupo.

Outro aspecto importante desses resultados é o fato de as variáveis defasadas não serem estatisticamente significantes. Isso mostra que a estabilidade das instituições que estão no grupo Top 5 é influenciada pelas variáveis correntes e não por ocorrências passadas, o que indica um ajuste rápido e de característica prospectiva nas previsões em adequação à realidade, e não um comportamento de inércia das previsões em relação a ocorrências anteriores. Isso não elimina a inércia na composição do grupo Top 5, cuja metodologia considera o erro em um conjunto móvel de períodos, de seis meses no caso do curto prazo, para diminuir o efeito de acerto aleatório na lista Top 5.

Uma estimação alternativa, utilizando o índice para a presença de uma instituição respondente em três meses consecutivos no grupo Top 5 não apresentou variáveis que fossem estatisticamente significativas na regressão, o que pode indicar que a presença em períodos mais longos no grupo com os menores erros é mais influenciada por características dessas instituições do que do cenário macroeconômico.

Apesar da proximidade da análise de recorrência com a análise do diferencial de desempenho de Prada (2007), os resultados não são diretamente comparáveis. A permanência no grupo Top 5 é associada por Prada (2007) ao esforço despendido nas previsões, com reflexo no resultado, enquanto a recorrência é associada ao erro de previsão e dispersão das previsões. Isso acaba por gerar a inclusão no grupo Top 5 de instituições mais hábeis com maior frequência. No período de 161 meses aqui considerado, 10 instituições aparecem no Top 5 entre 16 e 35 vezes, o que pode ser considerado um número superior ao que ocorreria se a presença fosse predominantemente aleatória entre os participantes. Por outro lado, 69 instituições aparecem em até quatro dos 161 meses, confirmando a heterogeneirdade entre os respondentes.

\section{Considerações finais}

O sistema de expectativas do $\mathrm{BCB}$ permite à autoridade monetária acompanhar em tempo real as alterações nas expectativas dos previsores profissionais que respondem ao sistema, permitindo a aferição de movimentos de convergência e divergência das expectativas. A importância do levantamento institucionalizado de expectativas do $\mathrm{BCB}$, entretanto, vai além da obtenção pela autoridade monetária de previsões qualificadas para variáveis macroeconômicas relevantes. Está também na divulgação sistemática dos resultados do levantamento, como ocorre com a distribuição às segundas-feiras do "Focus Relatório de Mercado", que tem ampla repercussão no noticiário econômico. 
Esse relatório divulga as previsões vigentes na sexta-feira da semana anterior. A repercussão das alterações nessas previsões, mesmo que fora da data de referência, ressalta a importância das informações coletadas. Percebe-se, com isso, que a pesquisa de expectativas do BCB é parte efetiva da estratégia de comunicação envolvida no sistema de metas de inflação. Dada essa importância, a estrutura de influência e imitação entre respondentes é uma decorrência esperada, como já detectado em Carvalho \& Minella (2012).

$\mathrm{O}$ fato de a data de referência ser importante para as previsões gera uma discussão teórica sobre o desenho do sistema de previsões. Como a data de referência é relevante, se o sistema de premiação considerasse um conjunto maior de dias, isso possivelmente geraria previsões potencialmente melhores. Isso decorreria de os respondentes serem induzidos a informarem à pesquisa as suas melhores previsões possíveis com maior frequência, em todos os dias no limite. O trade off decorrente, porém, envolve o custo gerado pela necessidade constante de revisão das previsões por parte dos respondentes, mesmo que não detectada essa necessidade pela análise do cenário econômico ou por demandas internas à própria insituição. Uma intuição possível para isso seria que o custo de atualização aumenta mais com o número de dias considerados do que a possível acurácia gerada pela revisão mais frequente, justificando a utilização de datas de referência e não intervalos. Por outro lado, custos marginais declinantes de efetuar revisões das previsões com maior frequência poderiam compensar esse efeito. A questão dos custos gerados aos respondentes pela pesquisa não é trivial, como discutido, por exemplo, em Stark (2013), o qual analisa o Survey of Professional Forecasters do Federal Reserve de Philadelphia. Esse artigo relata pesquisa junto aos respondentes sobre o esforço necessário ao fornecimento das previsões, horizonte de previsão e utilização das informações, mesmo sendo o levantamento trimestral. No sistema do BCB o custo do fornecimento das previsões pode ser ajustado até certo ponto pelo respondente, por não ser obrigatório informar todas as variáveis pesquisadas.

A frequência e magnitude dos ajustes nas expectativas, independente da sua informação ao sistema do BCB, é influenciada pelo custo de efetuar a revisão da previsão e o custo do erro da previsão. O custo desses erros pode advir, por exemplo, de sua influência sobre preços no mercado financeiro, em que uma previsão correta pode aumentar a possibilidade de ganhos, além do custo reputacional junto a clientes efetivos e em potencial.

Um aspecto a ser considerado no comportamento dos respondentes do sistema do $\mathrm{BCB}$ é o incentivo à acurácia das previsões, que é o objetivo do $\mathrm{BCB}$ com a premiação do Top 5. Como não há incentivo monetário direto para os premiados, o incentivo à obtenção da melhor previsão está associado a um ganho de reputação. Este pode ser tanto para o indivíduo responsável pela previsão, quanto para a instituição respondente, sendo frequentemente para ambos. As interações entre indivíduos e instituições que participam do survey do BCB podem ajudar a explicar questões como o fato de os participantes alterarem as suas previsões mesmo quando não há incentivo direto via efeito na classificação Top 5.

Para pesquisa futura, uma questão complementar que seria interessante de ser verificada é se os integrantes do grupo Top 5 realizam um maior número de alterações em comparação aos demais respondentes, para verificar diferenças na velocidade de incorporação de informações ou no grau de rigidez informacional entre os dois grupos, para o que será necessário analisar as séries de previsões desagregadas. 
Em horizontes mais longos do que os analisados neste artigo, as expectativas para diferentes variáveis interagirão, como no caso de inflação e taxa de juros, levantando a questão da causalidade entre as expectativas e o seu grau de rigidez. Isso envolve tanto o comportamento da economia, em que parece haver várias formas de rigidez nominal e real, quanto das expectativas, que também podem apresentar rigidez não necessariamente relacionada com a rigidez da variável. Nesse sentido, uma extensão deste artigo seria explorar, por exemplo, a chamada hipótese Friedman-Ball de relação entre taxa de inflação e incerteza nas previsões, seguindo a literatura baseada em Okun (1971), Taylor (1981), Ball (1992), Fountas (2001), entre outros.

\section{Agradecimentos}

Agradecemos a um parecerista anônimo da Revista pelas excelentes sugestões que permitiram melhorar o trabalho. Os erros remanescentes obviamente são de nossa responsabilidade.

Os autores agradecem o apoio do Conselho Nacional de Desenvolvimento Científico e Tecnológico - CNPq.

\section{Referências Bibliográficas}

Ball, L. (1992), 'Why does high inflation raise inflation uncertainty?', Journal of Monetary Economics 29(3), 371-388.

Banco Central do Brasil (2016), 'Sistema Expectativas de Mercado. (série perguntas mais frequentes)', Disponível em: http://www4.bcb.gov.br/pec/gci/port/focus/faq.asp. Consultado em $16 / 05 / 2017$.

Bordo, M. D. \& Siklos, P. L. (2017), 'Central bank credibility before and after the crisis', Open Economies Review 28(1), 19-45.

Bordo, M. \& Siklos, P. (2014), 'Central bank credibility, reputation and inflation targeting in historical perspective', Cambridge, MA: NBER. (NBER Working Paper W20693).

Branch, W. A. (2004), 'The theory of rationally heterogeneous expectations: evidence from survey data on inflation expectations', The Economic Journal 114(497), 592-621.

Caetano, S. M. \& Silva Júnior, G. E. (2012), 'Inflationary expectatives: predicting, persistence, and disagreement', Análise Econômica 30(58).

Cameron, A. C. \& Trivedi, P. K. (1990), 'Regression-based tests for overdispersion in the poisson model', Journal of econometrics 46(3), 347-364.

Carvalho, F. A. \& Minella, A. (2012), 'Survey forecasts in brazil: a prismatic assessment of epidemiology, performance, and determinants', Journal of International Money and Finance 31(6), 1371-1391.

Coibion, O., Gorodnichenko, Y. \& Kamdar, R. (2017), 'The Formation of Expectations, Inflation and the Phillips Curve', Cambridge, MA: NBER. (NBER Working Paper W23304). 
European Central Bank (2016), 'ECB Survey of Professional Forecasters - Background', Disponível em http://www.ecb.europa.eu/stats/prices/indic/forecast/html/index.en.html. Consultado em 28/06/2016.

Federal Reserve of Philadelphia (2016), 'Survey of Professional Forecasters - Documentation', Disponível em https://www.philadelphiafed.org//media/research-and-data/real-time-center/survey-of-professionalforecasters/spf-documentation.pdf?la=en. Consultado em 27/06/2016.

Fountas, S. (2001), 'The relationship between inflation and inflation uncertainty in the uk: 1885-1998', Economics Letters 74(1), 77-83.

Guillén, D. \& Garcia, M. (2014), 'Expectativas desagregadas, credibilidade do banco central e cadeias de markov', Revista Brasileira de Economia 68(2), 197-223.

Lamont, O. A. (2002), 'Macroeconomic forecasts and microeconomic forecasters', Journal of economic behavior E organization 48(3), 265-280.

Laster, D., Bennett, P. \& Geoum, I. S. (1999), 'Rational bias in macroeconomic forecasts', The Quarterly Journal of Economics 114(1), 293-318.

Lima, G. T., Setterfield, M. \& Silveira, J. J. (2014), 'Inflation targeting and macroeconomic stability with heterogeneous inflation expectations', Journal of Post Keynesian Economics 37(2), 255-279.

Mankiw, N. G., Reis, R. \& Wolfers, J. (2003), 'Disagreement about inflation expectations', NBER macroeconomics annual 18, 209-248.

Marques, A. B. C. (2012), 'Central Bank of Brazil's market expectations system: a tool for monetary policy', Bank for International Settlements 36, 304-324.

Okun, A. M. (1971), 'The mirage of steady inflation', Brookings Papers on Economic Activity 1971(2), 485-498.

Prada, M. (2007), 'Premiação Top 5: estudo de um caso de torneio no Brasil'. Dissertação, Mestrado em Economia, EESP-FGV-SP.

Rocha, M. V. (2010), 'Previsão da inflação no Boletim Focus: uma avaliação'. Dissertação de mestrado, EPGE - FGV/RJ.

Silveira, J. J. \& Lima, G. T. (2013), 'Regime monetário de meta de inflação em um ambiente de heterogeneidade de estratégias de formação de expectativas de inflação', Estudos Econômicos (São Paulo) 43(2), 213-239.

Stark, T. (2013), 'SPF panelists forecasting methods: a note on the aggregate results of a November 2009 special survey', Federal Reserve Bank of Philadelphia

Svensson, L. E. (1994), 'Estimating and interpreting forward interest rates: Sweden 1992-1994', Cambridge, MA: NBER. (NBER Working Paper W4871).

Taylor, J. B. (1981), 'On the relation between the variability of inflation and the average inflation rate', Carnegie-Rochester Conference Series on Public Policy 15, 57-85. 
Zarnowitz, V. \& Braun, P. (1993), Twenty-two years of the nber-asa quarterly economic outlook surveys: aspects and comparisons of forecasting performance, in 'IN: Stock, J. J., Watson, K. W., eds, Business cycles, indicators and forecasting', University of Chicago Press, pp. 11-94. 